\title{
A BROADCAST SYNTHESIZED FM MODULATOR WITH A RADIO DATA SYSTEM ENCODER
}

\author{
Jordi Berenguer-Sau, Member, IEEE and Angel Villanueva-Fernández \\ Department of Signal Theory and Communications \\ D3 Group - Electromagnetics \& Photonics Engineering \\ Polytechnic University of Catalonia (UPC) \\ Edifici D3, Campus Nord \\ C. Gran Capita, s/n, 08034-Barcelona, Spain \\ Phone: 34-3-401-59-06, Fax: 34-3-401-59-10 \\ e-mail: berengue@voltor.upc.es
}

\begin{abstract}
The design and development of a broadcast FM modulator with a RDS (Radio Data System) encoder is presented. The system is fully coherent with the modulated $R F$ frequency synthesized in the 87.5-108 $\mathrm{MHz}$ frequency band with $100 \mathrm{kHz}$ frequency steps. This feature has been obtained by a feedback loop where a PLL acts as a frequency demodulator, thus providing control capability on the phase noise of the modulated signal without any restrictions in the modulation bandwidth, thereby reducing the adjustments when a frequency change is made. The design of the $R D S$ encoder has been done according to the CCIR regulations, avoiding the BPSK approach, by means of current electronic circuits plus a switch-capacitor filter in a simplified implementation derived from the RDS standard and suitable for an eventual ASIC design. The developed system gives full capability as a FM stereo broadcast modulator according to the CCIR regulations, in order to be used in a university broadcast station for testing and developing all the features of the RDS system, specially the use of the transparent data channel as a communication way between the university community.
\end{abstract}

\section{INTRODUCTION}

The Radio Data System [1][2] is widely used in Europe in FM broadcast stations, but generally in a reduced form, providing only the main services related to the traffic and car security aspects. With the aim of testing and cleveloping all the possibilities of the RDS system in the context of a university FM station in order to use its capability for a low rate data transmission, we started to develop a FM modulator with a RIDS encoder with the requirement of an easy change of the modulation frequency in any channel of the 87.5-108 $\mathrm{MHz}$ band in addition to full system coherence.

The main difficulty found in the development of a RDS encoder was the lack of dedicated chips for this function, by contrast with the decoders whose commercial use in car radios and home receivers make these circuits widely available. In our design we have excluded the BPSK approach or the use of an expensive DSP for processing a very low rate (1200 bps) data signal; instead we built an encoder using current digital circuits plus a switch-capacitor filter, in an original implementation derived from the RDS standard, and suitable for an ASIC development.

In order to design the modulator in accordance to the CCIR regulations, the low frequency modulation of a crystal oscillator and its subsequent frequency multiplication was not considered due to its complexity in the RF hardware. Instead in order to achieve the 75 $\mathrm{kHz}$ frequency deviation with a baseband signal of $73 \mathrm{kHz}$ bandwidth we have choose the known feedback frequency Publisher Item Identifier: S 0018-9316(96)07162-4 stabilization system, but replacing the frequency discriminator in the feedback loop by a PLL thus improving the phase noise control of the modulated signal, in addition with a synthesized reference oscillator which provides the $100 \mathrm{kHz}$ steps and the frequency stability and phase noise characteristics of the output signal.

In this paper the implemented scheme of the RDS encoder and the analysis and experimental results of the whole synthesized FM modulator are presented.

\section{SYSTEM DESCRIPTION}

The block diagram of the proposed system is shown in Figure 1. The multiplex signal generator processes the Left and Right audio input channels; these signals, through a $50 \mu \mathrm{s}$ pre-accentuation filter are added giving the $(L+R)$ signal with a cutoff frequency of $15 \mathrm{kHz}$, and after subtraction the (L-R) signal is amplitude modulated with a suppressed carrier of $38 \mathrm{kHz}$ translating it to the $23-53 \mathrm{kHz}$ band; with the addition of the $19 \mathrm{kHz}$ subpilot signal and the consequent weighing process according to the CCIR regulations [3], the stereophonic multiplex signal is obtained. The $19 \mathrm{kHz}$ signal and its $38 \mathrm{kHz}$ harmonic are supplied from the frequency generator block.

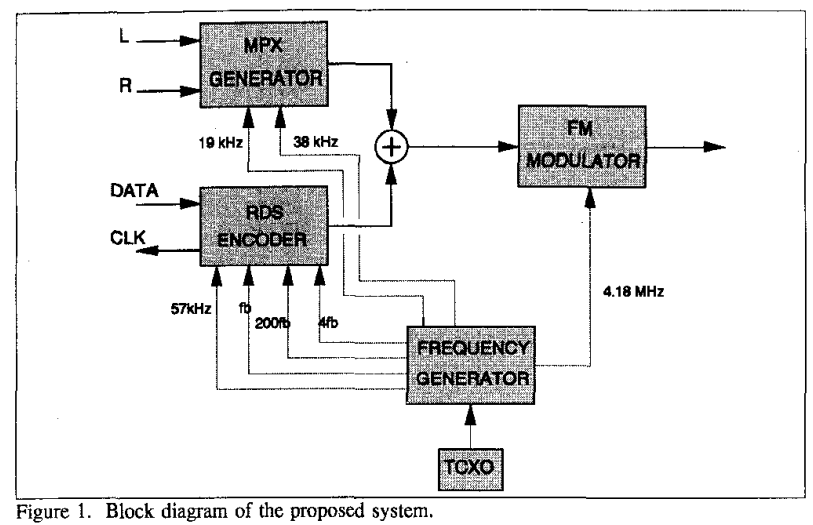

The RDS encoder generates a $1187.5 \mathrm{~Hz}$ clock signal for clocking the input serial data; this data signal is processed and coded and finally is amplitude modulated by a suppressed carrier of $57 \mathrm{kHz}$, translating it between $54.6 \mathrm{kHz}$ and $59.4 \mathrm{kHz}$. The obtained signal is then added to the previous multiplex audio signal in order to be used as modulation signal in the FM modulator. 
118

The FM modulator gives the RF carrier modulated by the multiplex and RDS signal in the FM band (87.5-108 MHz) with its frequency programmable in $100 \mathrm{kHz}$ steps. It includes a frequency synthesizer which gives a $82.5-103 \mathrm{MHz}$ signal used in the feedback loop as will be seen later.

The frequency generator block provides all the frequencies required by the rest of the modules. From a reference of $4.18 \mathrm{MHz}$ obtained from a TCXO which guarantees the frequency stability requirements [3], the $38 \mathrm{kHz}$ and $19 \mathrm{kHz}$ are obtained by direct division by 110 and 220 , respectively; the RDS carrier of $57 \mathrm{kHz}$ is obtained by frequency multiplication by 3 of the $19 \mathrm{kHz}$ signal using a PLL; from it, the bit rate of $1187.5 \mathrm{~Hz}\left(\mathrm{f}_{\mathrm{b}}\right)$ and the auxiliary frequencies of $4 \mathrm{f}_{\mathrm{b}}$ and $200 \mathrm{f}_{\mathrm{b}}$ are obtained by direct division by 48 and 12 , and frequency multiplication by 50 of the $4 f_{b}$ signal, respectively. Also the $4.18 \mathrm{MHz}$ frequency is used as a reference in the frequency synthesizer of the FM modulator, thus guarantying the full system coherence.

\section{THE RDS ENCODER}

Is well known that the Radio Data System is a supplementary data service which is added to the conventional multiplex signal of a FM broadcast station in order to provide the receivers several intelligent functions, specially to the radio-cars.

The RDS system was conceived as a digital evolution of the german analog system ARI, improving the classic traffic functions but introducing new services and functions as Radiotext or the Transparent Data Channel suitable for several applications. Although these functions and services are perfectly structured and well defined, at the present the Program Identifier (PI), Traffic Announcement (TA), list of Alternative Frequencies (AF) and the Traffic Program (TP), are the most used, being the others not usually implemented.

The codification standard of the RDS is shown in Figure 2. The main difficulty in its development arises in the design of the shaping filter with a transfer function:

$$
H_{T}(f)=\left\{\begin{array}{l}
\cos \frac{\pi f t_{d}}{4}, \text { for } 0 \leq f \leq \frac{2}{t_{d}} \\
0, \text { for } f>\frac{2}{t_{d}}
\end{array}\right.
$$

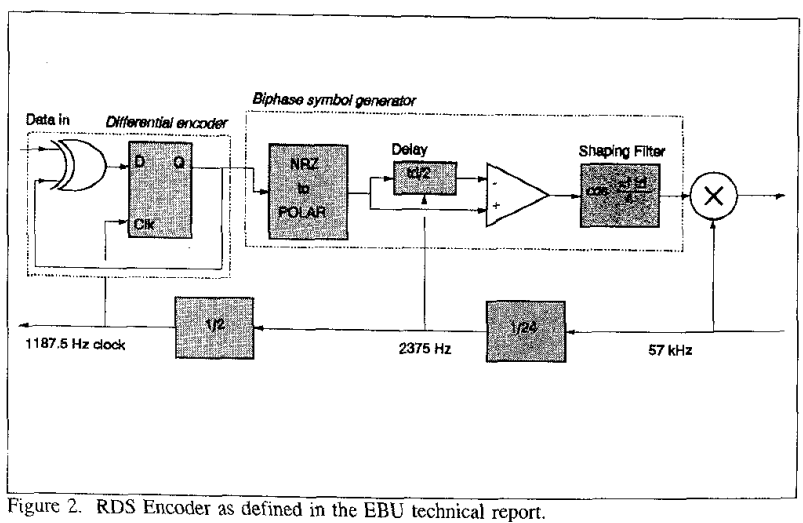

Probably the easiest, although not the best, implementation is with use of a DSP device, a fast device for a very low data stream (1187.5 bps!). This type of modulation is similar to a two level PSK modulation (BPSK) as the RDS standard specifies. For this reason some manufacturers choose this solution in order to build a costeffective encoder, based on commercial available PSK circuits.

The design method selected is very simple, and is based in the decomposition of the delayer+filter as follows [4].

The transfer function $\mathrm{H}_{\mathrm{T}}(\mathrm{f})$ shown in (1) can be expressed as the product of two transfer functions: a sinusoidal one $\mathrm{H}_{1}(\mathrm{f})$ and a rectangular filter $\mathrm{H}_{2}(\mathrm{f})$ as shown:

$$
H_{1}(f)=\cos \frac{\pi f t_{d}}{4}, \quad H_{2}(f)=\left\{\begin{array}{l}
1, \text { for } 0 \leq f \leq \frac{2}{t_{d}} \\
0, \text { for } f>\frac{2}{t_{d}}
\end{array}\right.
$$

The rectangular filter can be directly implemented by means of a switched-capacitor chip; instead for the simusoidal transfer function we have made an implementation based on its impulsional response $h_{1}(t):$

$$
h_{1}(t)=\frac{1}{2}\left[\delta\left(t+\frac{t_{d}}{8}\right)+\delta\left(t-\frac{t_{d}}{8}\right)\right]
$$

which after delaying the time origin to $t=t_{d} / 8$ can be rewritten as:

$$
h_{1}\left(t^{\prime}\right)=\frac{1}{2}\left[\delta\left(t^{\prime}\right)+\delta\left(t^{\prime}-\frac{t_{d}}{4}\right)\right]
$$

with this, the shaping filter can be implemented according to the Figure 3.

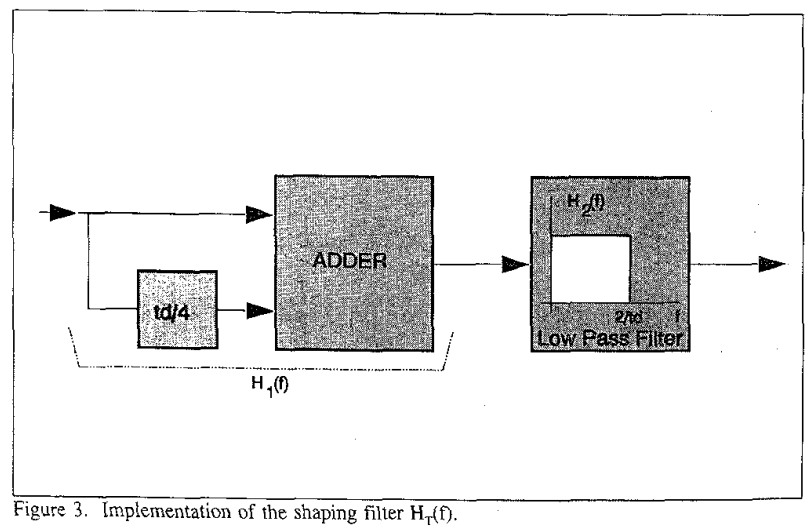

Analyzing the Biphase symbol generator of Figure 2, we obtain at the rectangular filter input for a logical " 1 " as data input the following:

$$
r\left(t^{\prime}\right)=\delta\left(t^{\prime}\right)+\delta\left(t^{\prime}-\frac{t_{d}}{4}\right)-\delta\left(t^{\prime}-\frac{t_{d}}{2}\right)-\delta\left(t^{\prime}-\frac{3 t_{d}}{4}\right)
$$

and for a logical "0" as data input:

$$
r\left(t^{\prime}\right)=-\delta\left(t^{\prime}\right)-\delta\left(t^{\prime}-\frac{t_{d}}{4}\right)+\delta\left(t^{\prime}-\frac{t_{d}}{2}\right)+\delta\left(t^{\prime}-\frac{3 t_{d}}{4}\right)
$$

and the Biphase Symbol Generator can be implemented as shown in Figure 4. 


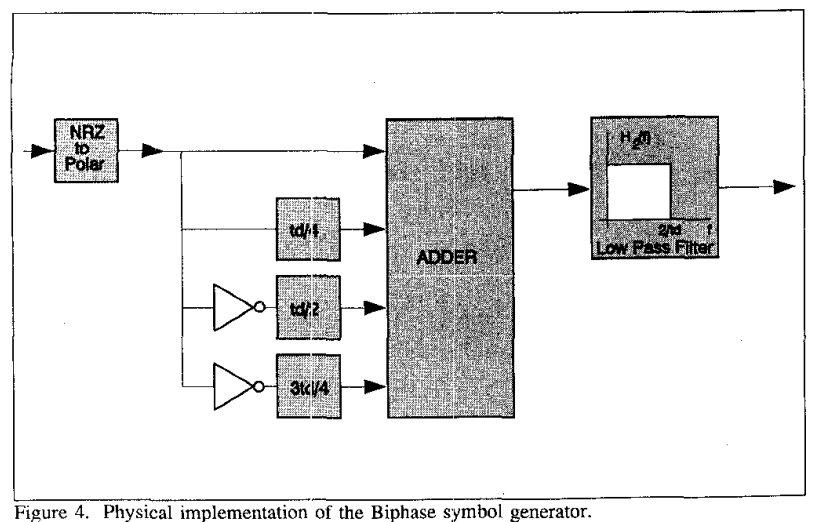

Although this structure is directly realizable, it presents some technological problems which can be avoided by doing the conversion from NRZ to polar impulses in two steps: a RZ to NRZ conversion before the delay process and the NRZ to polar impulse conversion after it, as shown in Figure 5. The digital signal at the input of the RZ to NRZ: converter implemented with operational amplifiers, is applied to the input of an analogical switches; these are operated by the output of a demultiplexer controlled by a binary counter synchronized to the bit rate $f_{b}$; the $\delta(t)$ function is approximately obtained from a monostable circuit which is applied at the demultiplexor input; as a result the ensemble operates as a sampler of a digital signal, thus obtaining at the rectangular filter input a stream of polar impulses.

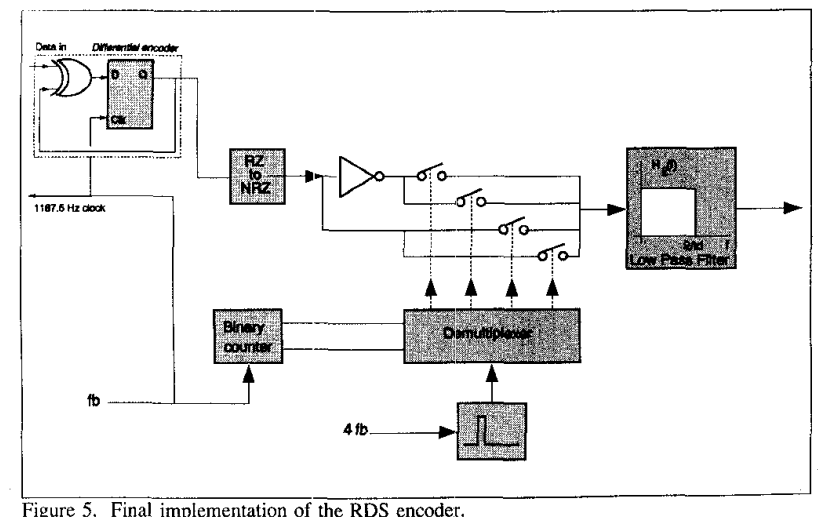

For the implementation of the rectangular filter we chose a switched capacitor 8th order elliptic filter MAX-293 from Maxims, whose cutoff frequency can be programmed by an external clock which a ratio frequency of $100: 1$, thus by means of an external signal of $200 \mathrm{f}_{\mathrm{b}}$ a cutoff frequency of $\left(\mathrm{t}_{\mathrm{d}} / 2\right)^{-1}$ is obtained keeping the full coherence of the system.

The signal obtained at the filter output of the encoder is shown in Figure 6 for a random input sequence, and after modulation in DSB by the $57 \mathrm{kHz}$ carrier we obtain the signal shown in Figure 7 .

\section{THE FREQUENCY MODULATOR}

In order to meet the frequency selection requirement of the RF output as any value ranging between 87.5 and $108 \mathrm{MHz}$ with a 100 $\mathrm{kHz}$ channel spacing, the solution based in a low frequency and low deviation frequency modulation in a VCXO and later frequency multiplication was not affordable. Also the use of a PLL as a frequency modulator was not possible due to the bandwidth of the multiplex signal, ranging between $20 \mathrm{~Hz}$ and $73 \mathrm{kHz}$.

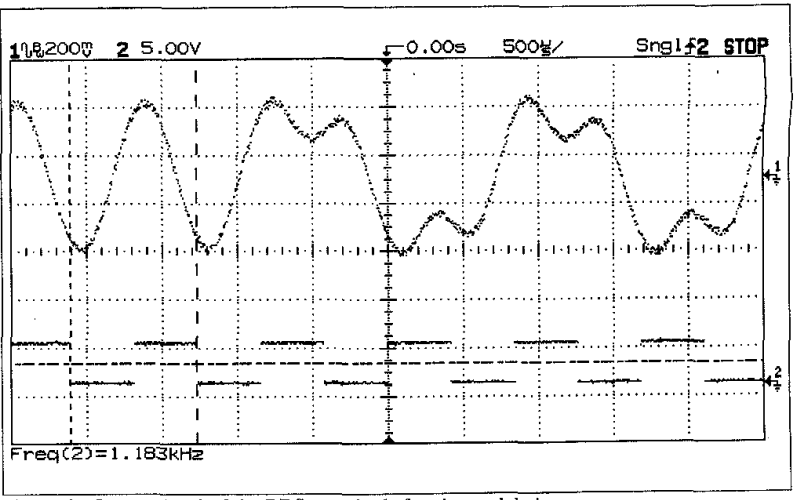

Figure 6. Output signal of the RDS encoder before its modulation.

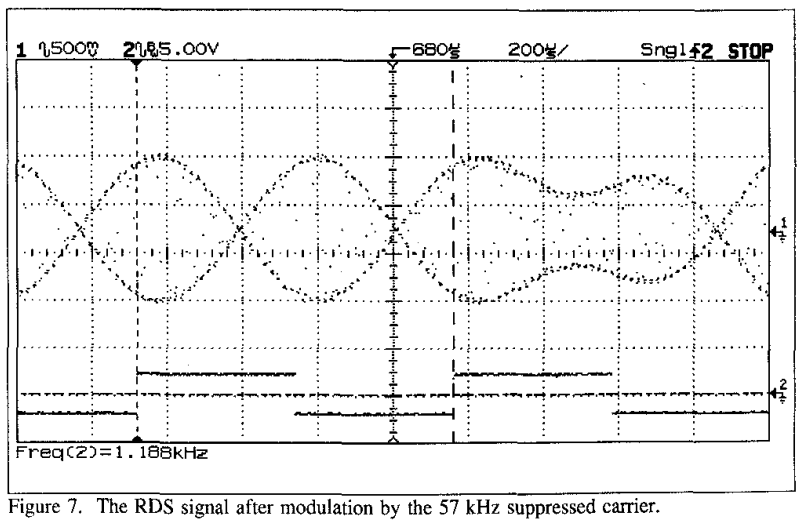

Finally, the adopted solution was the known frequency control loop [5], but replacing the frequency discriminator by a PLL, as shown in Figure 8.

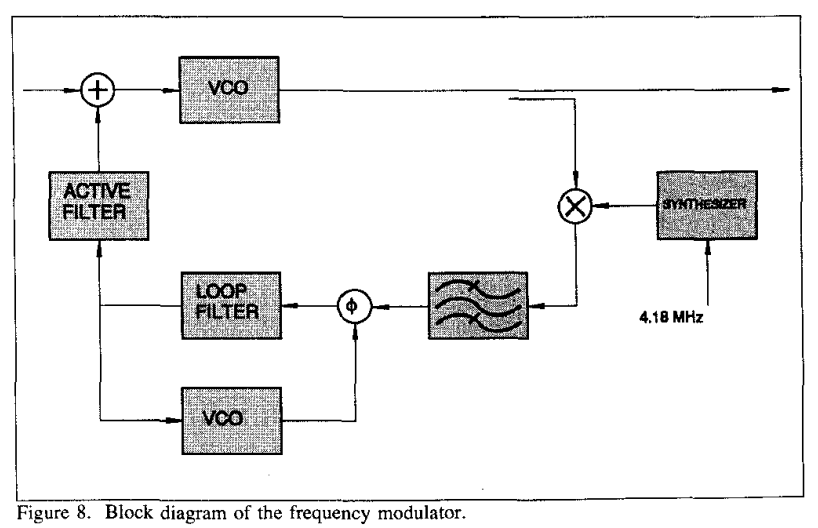

The output signal of a VCO which spans the 87.5-108 $\mathrm{MHz}$ bandwidth is connected through a directional coupler to the RF input of a mixer; as a local oscillator a frequency synthesizer obtained from the $4.18 \mathrm{MHz}$ reference is used, providing the $100 \mathrm{kHz}$ frequency steps in the $82.5-103 \mathrm{MHz}$ band. In this way a $5 \mathrm{MHz}$ intermediate frequency is obtained at the mixer output after bandpass filtering. Thus IF signal is then used as input to the PLL system, implemented with the NE564 circuit and designed with a reduced loop bandwidth; with this, the PLL removes the frequency modulation information of the input signal, but keeps the frequency drift of the RF VCO; the signal at the loop filter output is then applied to a low pass active filter which acts as a integrator, with its output added to the multiplex audio signal with the RDS signal, in 
120

order to be used as the control voltage of the $\mathrm{VCO}$, closing the feedback loop. With this arrangement, the phase noise and frequency stability of the $\mathrm{VCO}$ is improved by the frequency synthesizer through the feedback loop, but maintaining the required modulation bandwidth.

This scheme can be analyzed with help of the Laplace transform making use of the linear model shown in Figure 9, where the RF VCO and the PLL's VCO have been replaced by integrators of constants $K_{1}$ and $K_{2} \mathrm{rad} / \mathrm{s} / \mathrm{V}$, respectively, the mixer by a phase subtractor, the phase detector by another subtractor plus a constant of $\mathrm{K}_{\mathrm{d}} \mathrm{V} / \mathrm{rad}$, and the loop filter and the low-pass filter by its transfer functions $F(s)$ and $A(s)$, respectively. From Figure 9, the PLL can be modeled by its transfer function $\mathrm{H}_{1}(\mathrm{~s})$ obtained as:

$$
H_{1}(s)=\frac{V_{1}(s)}{\Theta_{o}(s)-\Theta_{r}(s)}=\frac{K_{d} F(s)}{1+K_{d} F(s) K_{2} / s}=\frac{K_{d}}{\tau_{1}} \frac{s\left(1+\tau_{2} s\right)}{s^{2}+\frac{K_{d} K_{2}}{\tau_{1}}\left(1+\tau_{2} s\right)}
$$

with the loop filter transfer function defined as:

$$
F(s)=\frac{1+\tau_{2} s}{\tau_{1} s}
$$

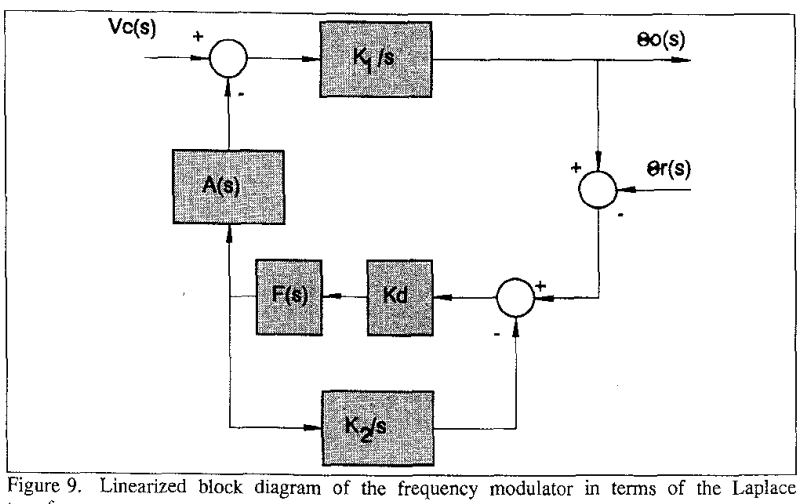
Figure 9.
transform.

Applying superposition, the output phase $\Theta_{0}(s)$ can be obtained as the sum of two transfer functions $M_{1}(s)$ and $M_{2}(s)$ from the contributions of the modulation signal $\mathrm{V}_{c}(\mathrm{~s})$ and the reference oscillator phase $\Theta_{\mathrm{r}}(\mathrm{s})$ :

$$
\begin{gathered}
M_{1}(s)=\frac{\Theta_{o}(s)}{V_{c}(s)}=\frac{K_{1}\left(s^{2}+K_{d} K_{2} \frac{\tau_{2}}{\tau_{1}} s+\frac{K_{d} K_{2}}{\tau_{1}}\right)}{s\left(s^{2}+K_{d} \frac{\tau_{2}}{\tau_{1}}\left(K_{2}+A(s)\right)+\frac{K_{d}}{\tau_{1}}\left(K_{2}+A(s)\right)\right)} \\
M_{2}(s)=\frac{\Theta_{o}(s)}{\Theta_{r}(s)}=\frac{K_{1} K_{d}}{\tau_{1}} \frac{A(s)\left(1+\tau_{2} s\right)}{s^{2}+\left(1+\tau_{2} s\right)\left(\frac{K_{d} K_{2}}{\tau_{1}}+K_{1} A(s)\right)}
\end{gathered}
$$

and after replacing the low-pass filter by its imperfect integrator transfer function:

$$
A(s)=\frac{1+\tau_{4} s}{\tau_{3} s}
$$

we finally obtain:

$$
M_{1}(s)=\frac{K_{1}\left(s^{2}+K_{d} K_{2} \frac{\tau_{2}}{\tau_{1}} s+\frac{K_{d} K_{2}}{\tau_{1}}\right)}{s^{3}+K_{d} \frac{\tau_{2}}{\tau_{1}}\left(K_{2}+\frac{\tau_{4}}{\tau_{3}}\right) s^{2}+\frac{K_{d}}{\tau_{1}}\left(\frac{1}{\tau_{3}}+K_{2}+\frac{\tau_{4}}{\tau_{3}}\right) s+\frac{K_{d}}{\tau_{1} \tau_{3}}}(\mathbf{1 2 )}
$$

Figures 10 and 11 show the Bode plots of both functions where the values $\mathrm{K}_{1}=18.8610^{6} \mathrm{rad} / \mathrm{s} / \mathrm{V}, \mathrm{K}_{\mathrm{d}}=0.3 \mathrm{~V} / \mathrm{rad}, \mathrm{K}_{2}=12.510^{6} \mathrm{rad} / \mathrm{s} / \mathrm{V}$, $\tau_{3}=1000 \tau_{4}$ and a damping coefficient for the PLL of $\zeta=1.14$ were assumed; also a $500 / 2 \pi \mathrm{Hz}$ and a $10 / 2 \pi \mathrm{Hz}$ values were selected for the loop filter bandwidth and the zero of the $\mathrm{A}(\mathrm{s})$ function, respectively. As it can be shown, the $\mathrm{M}_{\mathrm{I}}(\mathrm{s})$ acts as a low pass-filter, keeping the integrator behavior of the $\mathrm{VCO}$, not limiting the modulation voltage; instead, the $\mathrm{M}_{2}(\mathrm{~s})$ has a characteristic low pass transfer function where its first zero $(10 \mathrm{rad} / \mathrm{s})$ is the $\mathrm{A}(\mathrm{s})$ zero and the second pole frequency $(500 \mathrm{rad} / \mathrm{s})$ is the loop filter cutoff frequency; by adjusting these two parameters the phase noise of the output signal is controlled.

If we analyze the transfer function of the original system with the frequency discriminator instead of the PLL, after modelling it as a derivator of constant $\mathrm{K} \mathrm{V} / \mathrm{rad} / \mathrm{s}$ in terms of Laplace transform, and replacing the $\mathrm{A}(\mathrm{s})$ filter by an amplifier of value $\mathrm{A}$, the system transfer functions is reduced to:

$$
M_{1}(s)=\frac{K_{1}}{s\left(1+A K_{1} K\right)} \propto \frac{1}{s A K} ; \quad M_{2}(s)=\frac{K A K_{1}}{1+A K K_{1}} \approx 1
$$

where the $M_{1}(s)$ has been simplified to an integrator (the VCO behavior) but with a smaller constant than the original $K_{1}$, and the $\mathrm{M}_{2}(\mathrm{~s})$ approaches to the unity, not allowing any control on the phase noise of the main VCO.

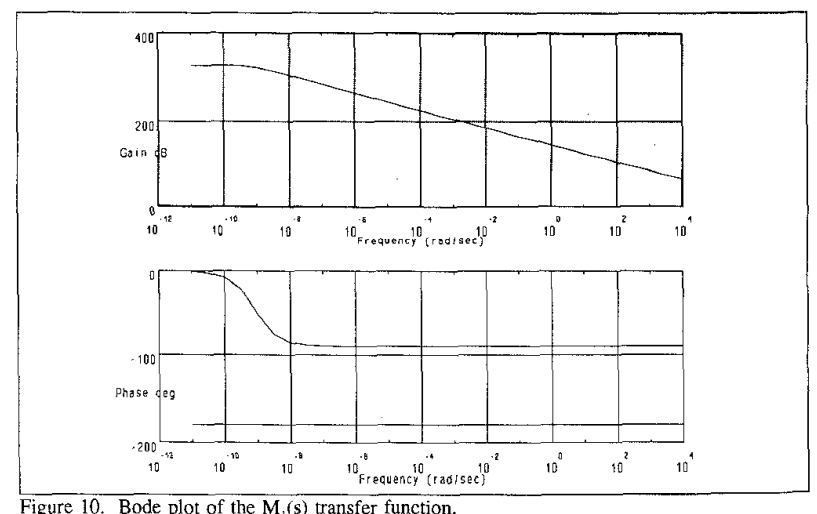

Figure 10 . Bode plot of the $\mathrm{M}_{1}(\mathrm{~s})$ transfer function. 


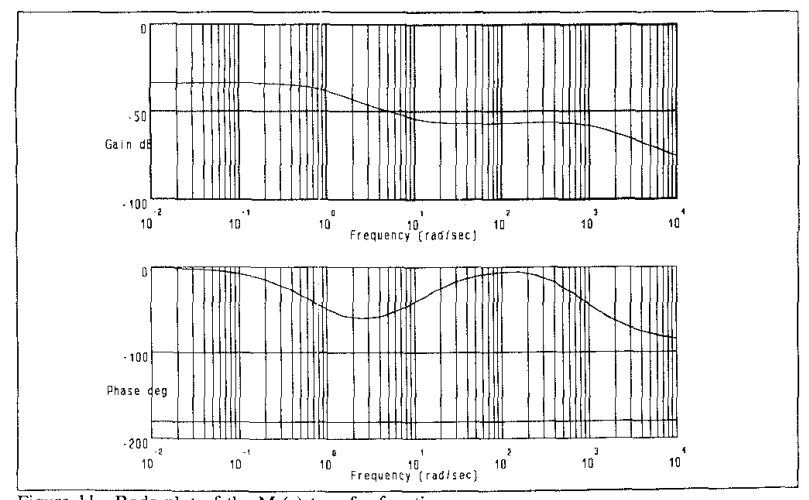

Figure 11. Bode plot of the $\mathrm{M}_{2}(\mathrm{~s})$ transfer function.

\section{CONCLUSIONS}

A RDS encoder suitable for its use in a broadcast FM station has been presented. Its conception is derived from the arrangement of the original RDS standard, where the conforming filter has been replaced by a rectangular switched-capacitor filter plus digital circuitry, avoiding the BPSK approach.

A synthesized FM modulator has been also presented. The frequency synthesis requirement has been performed by means of a feedback loop with a PLL acting as a frequency demodulator, showing the improvement on the phase noise characteristics of the modulated signal.

With them, a stereophonic synthesized FM modulator with a RDS encoder has been made. It will be used for testing the RDS functions an to develop a transparent data channel application in a University FM Station, for its use as a low rate data transmitter to the student community of the university informations.

\section{ACKNOWLEDGMENTS}

The authors wish to thank Professor Lluis Jofre for his support in the development of this project.

\section{REFERENCES}

[1].- EBU Technical Centre, "Specifications of the Radio Data System RDS for VHF/FM Sound Broadcasting." Tech. 3244E. Belgium, 1984.

[2].- CCIR, "Automatic Tuning System and other applications in FM Broadcast Fieceivers for its use in a Pilot Frequency System." Annex I, Rc. 643-1.

[3].- CCIR, "Transmission regulations for VHF/FM Sound Broadcasting." Rec. 450-1.

[4].- H. Taub and D. Schilling, Principles of Communication Systems. Singapore: McGraw-Hill, 1987, pp. 304-310.

[5].- K. Clarke and D. Hess, Communication Circuits: Analysis and Design. Krieger Publishing, Malabar, 1994, pp. 562-565.

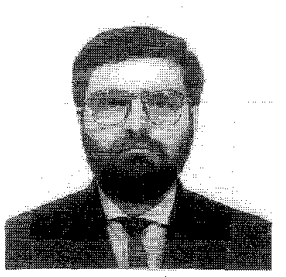

Jordi Berenguer i Sau (S'83-M'88), was born in Manresa, Spain in 1959. He received the Telecommunication Engineer and the Doctor Engineer on Telecommunication degrees in 1984 and 1988, respectively, both from the Telecommunication Engineering School of Barcelona of the Polytechnic University of Catalonia (UPC). In 1988 he joined the company Mier Comunicaciones as head of the RF laboratory working in the design of frequency synthesizers for TV transponders, and opening a new activity on the design of MMIC components. In 1992 he joined to the Electromagnetics and Photonics Engineering Group of the Signal Theory and Communications Department of the UPC as a Associated Professor as a responsible of the design and teaching activities of the Communications Laboratories of the Universitary Polytechnic School of the Baix Llobregat (UPC). His main research activities are with the design of microwave and RF subsystems, microwave frequency synthesis, and RF circuitry for broadcast applications. Actually his activity is centered in the design of a data transmission system using RDS, and the design of linearizers for RF power amplifiers.

Dr. Berenguer was awarded twice times by the Spanish Telecommunication Engineers Association by his Final Career Project (1985) and his Doctoral Thesis on microwave PLL's (1989).

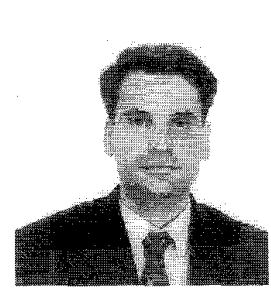

Ángel Villanueva Fernández, was born in Barcelona, Spain in 1966. He received the Telecommunication Engineer degree in 1995 from the Telecommunication Engineering School of Barcelona of the Polytechnic University of Catalonia (UPC). His professional activity started in 1984 as a Telecommunication Specialist in the Spanish National Railway Company (RENFE) until 1995. In January 1996 he joined to the IDOM Engineering and Consulting company as a project engineer.

Mr. Villanueva received in October 1995 the spanish Retevisión Award to the best project in the field of new techniques in image and sound broadcasting. 